1996

\title{
Nonlinear Control of Dynamical Systems from Time Series
}

Valery Petrov

Kenneth Showalter

Follow this and additional works at: https://researchrepository.wvu.edu/faculty_publications

\section{Digital Commons Citation}

Petrov, Valery and Showalter, Kenneth, "Nonlinear Control of Dynamical Systems from Time Series" (1996). Faculty Scholarship. 247. https://researchrepository.wvu.edu/faculty_publications/247 


\title{
Nonlinear Control of Dynamical Systems from Time Series
}

\author{
Valery Petrov and Kenneth Showalter* \\ Department of Chemistry, West Virginia University, Morgantown, West Virginia 26506-6045
}

(Received 20 September 1995)

\begin{abstract}
Feedback control of multidimensional, nonlinear single-input single-output systems is formulated in terms of an invariant hypersurface in the delayed state space of a system observable and a control parameter. The surface is created directly from the response of the system to random perturbations, providing a model-independent nonlinear control algorithm. The algorithm can be used to stabilize unstable states or to drive a system to any particular objective state in a minimum number of steps. [S0031-9007(96)00095-6]
\end{abstract}

PACS numbers: 05.45.+b, 82.40.Bj

The OGY (Ott-Grebogi-Yorke) [1] method for stabilizing unstable periodic orbits initiated a flurry of theoretical developments and experimental applications of feedback control to nonlinear dynamical systems [2-11]. Recent advances in control of high-dimensional systems offer new possibilities for manipulating complex temporal and spatiotemporal behavior [12-14]. All of these methods, however, are based on linearized models and the feedback control is therefore restricted to small perturbations in the linear regime. Here we present a new, integrated approach for nonlinear feedback control, where the response of the system to random perturbations is used directly to construct the control law as a multidimensional surface in the time-delayed space.

We demonstrate the approach with the Gray-Scott model for cubic autocatalysis in a flow reactor [15]. The governing dimensionless equations have the form

$$
\begin{aligned}
& \partial \alpha / \partial t=(1-\alpha) / T_{\text {res }}-\alpha \beta^{2}, \\
& \partial \beta / \partial t=\left(\beta_{0}-\beta\right) / T_{\text {res }}+\alpha \beta^{2}-\kappa_{2} \beta .
\end{aligned}
$$

With $\beta_{0}=\frac{1}{15}, \kappa_{2}=0$, the model is one-dimensional and displays one unstable and two stable stationary states over the range of reciprocal residence time $1 / T_{\text {res }}=$ $0.23-0.35$. Transitions from one stable state to the other can be induced by applying appropriate perturbations to $1 / T_{\text {res. }}$. Perturbations can also move the system to the unstable stationary state, but it will relax back to one of the stable states unless some form of feedback stabilization is applied.

We now describe how to control transitions between the stable and unstable states using a nonlinear control surface constructed from time series. For a one-dimensional system, the control surface is constructed by observing the transitions from an initial state $x_{I}(t)$ to a final state $x_{F}(t+\tau)$ that result from the application of perturbation $p$ during the sampling interval $\tau$. The collected triplets of values $\left(x_{I}, x_{F}, p\right)$ lie on a surface in a three-dimensional space. This nonlinear surface,

$$
p_{I \rightarrow F}=C\left(x_{I}, x_{F}\right),
$$

defines the perturbation that moves the system from an initial state $x_{I}$ to a desired final state $x_{F}$ in one iteration. Even though the identification stage can produce only a finite number of points, linear interpolation between closest neighbors can be used to construct the remainder of the surface.

Figure 1 shows the control surface for (1) generated from a series of responses to sequential random perturbations. The solid (open) circle corresponds to a transition from a stable (unstable) state to the coexisting unstable (stable) state in the region of bistability. Figure 2 shows a time series with transitions between the stable and unstable states. A similar procedure can be used for targeting and stabilizing fixed points in 1D return maps.

The control of multidimensional systems is more challenging since the initial and final states of (2) are no longer defined by the readings before and after the sampling interval. To derive an analogue of the control surface for such systems we first consider a linear twodimensional model and then generalize to include extra dimensions and nonlinear terms. The time discretized behavior of a linear two-variable system around a stationary state can be decomposed into the motion along the

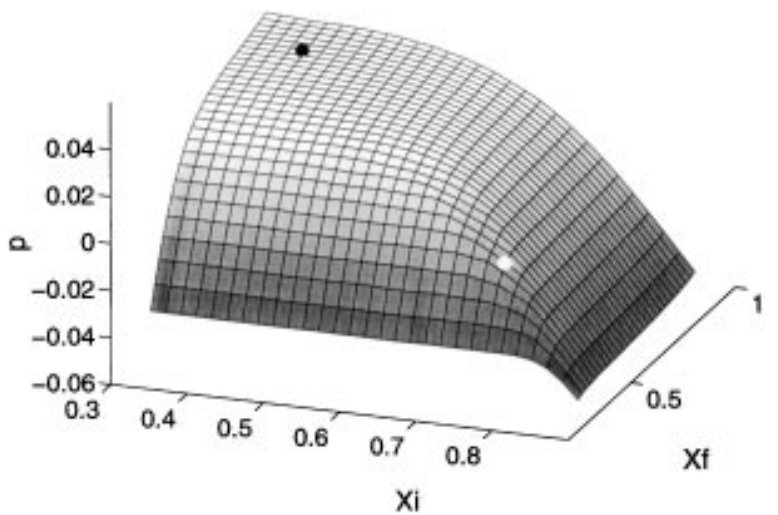

FIG. 1. Control surface for time-discretized $(\tau=5.0)$ onedimensional bistable system (1) at $1 / T_{\text {res }}=0.3$. The perturbation $p_{i}=1 / T_{\text {res }}-0.3$ and the observable $x_{i}=\alpha\left(t_{i}\right)$. Solid circle corresponds to transition from a stable state $\left(x_{I}=0.41\right)$ to the unstable state $\left(x_{F}=0.76\right)$; open circle shows perturbation required to move the system from the unstable to the stable state (in one iteration). 

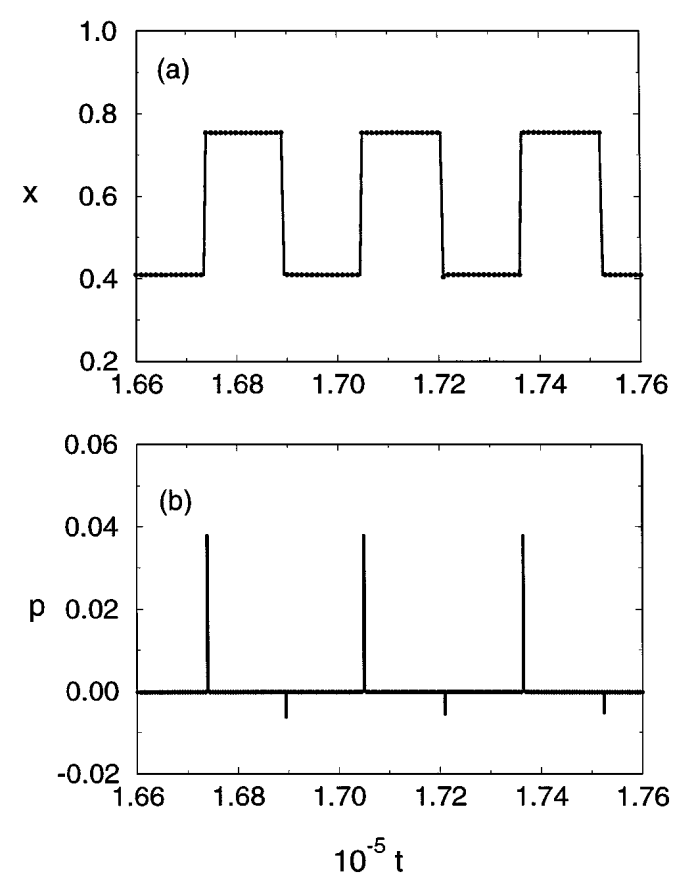

FIG. 2. (a) Time series of the one-dimensional bistable system (1) as it is moved from the stable state to the unstable state and back again; (b) applied perturbations.

eigenvectors $\xi$ and $\eta$ :

$$
\begin{aligned}
& \xi_{i+1}=\lambda_{\xi} \xi_{i}+\left(1-\lambda_{\xi}\right) \alpha_{\xi} p_{i+1}, \\
& \eta_{i+1}=\lambda_{\eta} \eta_{i}+\left(1-\lambda_{\eta}\right) \alpha_{\eta} p_{i+1},
\end{aligned}
$$

where $\lambda_{\xi}, \lambda_{\eta}$ are the eigenvalues along the corresponding eigenvectors, and $\alpha_{\xi}=\partial \xi_{S} / \partial p$ and $\alpha_{\eta}=\partial \eta_{S} / \partial p$ are the shifts of the stationary state arising from the applied perturbation. The perturbation is constant during the iteration, and when $p=0$ the stationary state is at the origin.

We assume the availability of only one observable on which the system dynamics is projected with some coefficients $t_{\xi}$ and $t_{\eta}$ :

$$
x_{i}=t_{\xi} \xi_{i}+t_{\eta} \eta_{i}
$$

If the system is initially at the state $\xi_{i}, \eta_{i}$, the next observations of $x$ will then be defined by Eqs. (3) and (4), with $x_{i+1}$ being a linear combination of $\xi_{i}, \eta_{i}$, and $p_{i+1}$,

$$
x_{i+1}=\lambda_{\xi} t_{\xi} \xi_{i}+\lambda_{\eta} t_{\eta} \eta_{i}+A p_{i+1},
$$

and $x_{i+2}$, which also includes $p_{i+2}$,

$$
x_{i+2}=\lambda_{\xi}^{2} t_{\xi} \xi_{i}+\lambda_{\eta}^{2} t_{\eta} \eta_{i}+B p_{i+1}+A p_{i+2},
$$

where

$$
\begin{aligned}
& A=\left(1-\lambda_{\xi}\right) \alpha_{\xi} t_{\xi}+\left(1-\lambda_{\eta}\right) \alpha_{\eta} t_{\eta}, \\
& B=\left(1-\lambda_{\xi}\right) \lambda_{\xi} \alpha_{\xi} t_{\xi}+\left(1-\lambda_{\eta}\right) \lambda_{\eta} \alpha_{\eta} t_{\eta} .
\end{aligned}
$$

It follows from (4) and (5) that the state of the system (i.e., the coordinates along the system manifolds) at iteration $i$ or $i+1$ can be reconstructed from two time-delayed readings of the observable and the perturbation applied to the system. Analogous arguments for an $m$-dimensional sys- tem define the state as a linear combination of $m$ delayed observations and $m-1$ perturbations:

$$
\begin{aligned}
& \left(\xi_{i}, \eta_{i}, \ldots\right) \\
& =L_{D}\left(x_{i}, x_{i-l}, \ldots, x_{i-m+1} ; p_{i}, p_{i-1}, \ldots, p_{i-m+2}\right),
\end{aligned}
$$

where $L_{D}$ is a linear function.

Once the system state is known, the control perturbations can be applied to direct the system to a desired objective state. We assume that only one control parameter is available to alter the system dynamics. From the second iteration of (3),

$$
\begin{aligned}
& \xi_{i+2}=\lambda_{\xi}^{2} \xi_{i}+\left(1-\lambda_{\xi}\right) \alpha_{\xi}\left(\lambda_{\xi} p_{i+1}+p_{i+2}\right), \\
& \eta_{i+2}=\lambda_{\eta}^{2} \eta_{i}+\left(1-\lambda_{\eta}\right) \alpha_{\eta}\left(\lambda_{\eta} p_{i+1}+p_{i+2}\right),
\end{aligned}
$$

we see that two perturbations, $p_{i+1}$ and $p_{i+2}$, can move the system from any initial state $\left(\xi_{i}, \eta_{i}\right)$ to any final state $\left(\xi_{i+2}, \eta_{i+2}\right)$ provided that $\lambda_{\xi} \neq \lambda_{\eta}, \lambda_{\xi}, \lambda_{\eta} \neq 1$ and $\alpha_{\xi}, \alpha_{\eta} \neq 0$ [13]. The control perturbations are a linear combination of the initial and final states. Even though a sequence of two perturbations must be applied before the desired state is reached, it is necessary to determine only the first perturbation explicitly, since the second is calculated using the same expression at the next iteration with the updated readings. For the linear $m$-dimensional system, the control algorithm is written as

$$
p_{i+1}=L_{C}\left(\left(\xi_{i}, \eta_{i}, \ldots\right),\left(\xi_{i+m}, \eta_{i+m}, \ldots\right)\right),
$$

where $L_{C}$ is a linear function. Such a function will always exist provided that the system is controllable and observable, i.e., $\lambda_{j} \neq \lambda_{k}$ for $j \neq k, \lambda_{j} \neq 1$ and $\alpha_{j}, t_{j} \neq 0$ for all $j$.

The sequence of $m$ readings $x$ and $m-1$ perturbations $p$ can be utilized in (8) to realize the final state in (10). It will not be apparent to the observer, however, that the system has reached that state until the $m$ th iteration. It is therefore convenient to define the objective state in a form independent of the control perturbations $p_{i+1}, \ldots, p_{m}$. With this in mind, we consider two separate control problems: stabilizing unstable states and attaining a prescribed constant output. In each, the objective state is realized in a minimum number of steps.

With no external perturbations, stationary state behavior is characterized by the absence of motion, i.e., $x_{i+1}-x_{i}=$ 0 and $p_{i}=0$ for $i=1, \ldots, m$. The difference between readings for consecutive steps in the two-variable system can be written by subtracting (6), (5) and (5), (4):

$$
\begin{aligned}
x_{i+1}-x_{i}= & \left(\lambda_{\xi}-1\right) t_{\xi} \xi_{i}+\left(\lambda_{\eta}-1\right) t_{\eta} \eta_{i}+A p_{i+1}, \\
x_{i+2}-x_{i+1}= & \lambda_{\xi}\left(\lambda_{\xi}-1\right) t_{\xi} \xi_{i}+\lambda_{\eta}\left(\lambda_{\eta}-1\right) t_{\eta} \eta_{i} \\
& +(B-A) p_{i+1}+A p_{i+2} .
\end{aligned}
$$

It follows that the system state can be determined as a solution of (11) from the two differences in readings and two perturbations. For an $m$-dimensional system, the state 
can be determined from $m$ consecutive differences and $m$ applied perturbations:

$$
\begin{array}{r}
\left(\xi_{i+m}, \eta_{i+m}, \ldots\right) \\
=L_{S}\left[\left(x_{i+m+1}-x_{i+m}\right), \ldots,\left(x_{i+2 m}-x_{i+2 m-1}\right) ;\right. \\
\left.p_{i+m+1}, \ldots, p_{i+2 m}\right] .
\end{array}
$$

Equation (12) has a convenient form for defining a stationary state or fixed point since no other information is required for the corresponding position in phase space.

Combining (8) and (12) with (10) yields a general expression for the stabilization of unstable states in an $\mathrm{m}$ dimensional system:

$$
\begin{array}{r}
p_{i+1}=S\left[x_{i}, x_{i-1}, \ldots, x_{i-m+1} ; p_{i}, p_{i-1}, \ldots, p_{i-m+2} ;\right. \\
\left(x_{i+m+1}-x_{i+m}\right), \ldots,\left(x_{i+2 m}-x_{i+2 m-1}\right) ; \\
\left.p_{i+m+1}, \ldots, p_{i+2 m}\right],
\end{array}
$$

where $S$ is the system invariant function. In the linear region, $S$ can be identified from the time series of the randomly perturbed system by solving the associated set of linear equations with $4 m$ unknown coefficients. We assume that Eq. (13) can be expanded into the nonlinear region with the system state determined by the coordinates on the curvilinear stable and unstable manifolds replacing the corresponding eigenvectors. Nonlinear terms can be incorporated through multivariable Fourier series expansion or by creating a nonlinear surface in $4 m$-dimensional space using linear interpolation between nearest neighbors. Neural networks that are suited for fitting nonlinear functions can also be used to learn the $S$ function on the basis of available data sets. Once constructed, the $S$ function is a control invariant for a particular system that can be used to target unstable states from anywhere in phase space provided the perturbations do not exceed limits imposed by the system dynamics and that the function remains single valued. The convergence of the data points to a single-valued function provides a criterion for system controllability in the nonlinear sense. In some cases, however, the application of the $S$ function is ambiguous; for example, three different steady states are present for $p=0$ in the bistable region. Additional restrictions, such as limiting the range of the bifurcation parameter to single-valued regions can be imposed, or, alternatively, one can use the $G$ function described below to target a particular state.

The process of stabilization is carried out as follows: The $m$ delayed readings and $m-1$ delayed perturbations that define the current state are substituted into the first set of terms in the $S$ function [upper line of (13)]. The desired behavior yields zeros for the second set of terms in $S$. With these substitutions, the $S$ function returns the first control perturbation. The second control perturbation is returned on the next iteration, and so on. After completion of the $m$-perturbation cycle, the system will reside very close to the objective state.

Unstable periodic orbits or stationary states can be tracked as a bifurcation parameter is slowly varied [16-
18]. It may be desirable, however, for the system to reach some prescribed objective state rapidly. Thus we now consider how the system can be moved in $m$ perturbations from any point to a desired point where the observable has a constant value $g$. It is not necessary to know the exact value of the bifurcation parameter at this point if the final state in (10) is defined by $p_{i+1}-p_{i}=0$ and $x_{i}=g$ for $i=1, \ldots, m$ and $g$ corresponds to a state existing in the bifurcation diagram. It is possible to reconstruct the state of the two-dimensional system, for example, from three consecutive observations of $x$ and one difference in $p$ using (4), (5), and (6). In general, $m$-dimensional systems require $m+1$ readings of $x$ and $m-1$ perturbation differences for the reconstruction:

$$
\begin{aligned}
& \left(\xi_{i+m}, \eta_{i+m}, \ldots\right)=L_{G}\left[x_{i+m}, \ldots, x_{i+2 m} ;\right. \\
& \left.\left(p_{i+m+2}-p_{i+m+1}\right), \ldots,\left(p_{i+2 m}-p_{i+2 m-1}\right)\right] .
\end{aligned}
$$

The appropriate control surface $G$ for driving the system output to some objective value is then constructed by combining (8), (10), and (14):

$$
\begin{gathered}
p_{i+1}=G\left[x_{i}, x_{i-1}, \ldots, x_{i-m+1} ; p_{i}, p_{i-1}, \ldots, p_{i-m+2} ;\right. \\
x_{i+m}, \ldots, x_{i+2 m} ; \\
\left.\left(p_{i+m+2}-p_{i+m+1}\right), \ldots,\left(p_{i+2 m}-p_{i+2 m-1}\right)\right] .
\end{gathered}
$$

The $G$ function can be identified from the system response in a fashion similar to the $S$ function identification. The control perturbation is returned by the $G$ function when the second set of terms [middle line of (15)] yields $x_{i+m}, \ldots, x_{i+2 m}=g$ and the perturbation differences are set to zero. The effective system dimension $m$ is usually not known in advance. Following methods developed for linear control [13], different values of $m$ can be used for creating the control surface and the fitting error is then evaluated. The value of $m$ that yields the minimum error is selected for control.

We now demonstrate stationary state stabilization and targeting objective states with the two-variable Gray-Scott model, where the parameter $\kappa_{2}=\frac{1}{40}$. The model exhibits a Hopf bifurcation at $1 / T_{\text {res }}=0.0049$. Changes in $1 / T_{\text {res }}$ from 0.0049 to 0.00508 and back again move the system from one value of the stationary state to another, as shown in Fig. 3. The oscillatory transients exhibited by the autonomous system arise from the slowing down in the vicinity of the Hopf bifurcation. The solid line shows the tracking obtained by use of the $G$ function. The linear version of the algorithm works well in this region since the variations are small. As shown in Fig. 3, only two iterations are necessary to move the system between the two stationary state values.

The autonomous Gray-Scott system displays nonlinear relaxation oscillations with $1 / T_{\text {res }}=0.0037$. The sevendimensional nonlinear $S$ and $G$ surfaces were obtained for these conditions by applying 1000 random perturbations to the system parameter $1 / T_{\text {res }}$. Each surface was constructed using linear interpolation from 8 neighboring data 

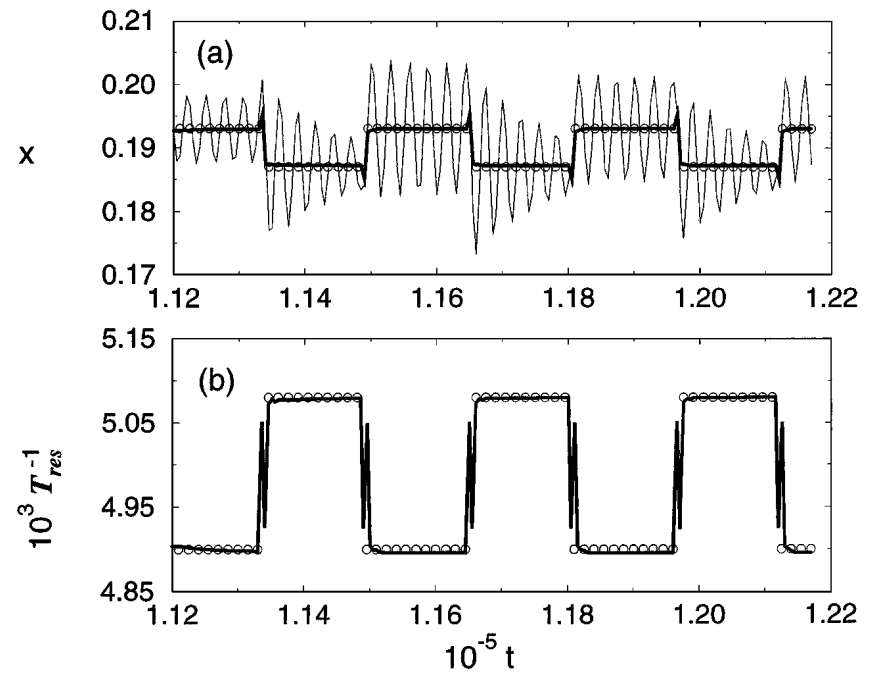

FIG. 3. (a) Response of autonomous (- ${ }^{-}$) and controlled ( in the control parameter move the system between two

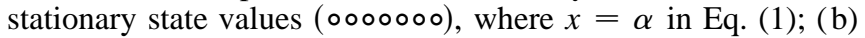
corresponding variations of $p=1 / T_{\text {res }}$.

points in the phase space. The system converges to the stationary state upon activation of the control algorithm. The convergence rate is initially slow, however, due to the sparseness of the control surface and the restriction imposed by the system dynamics on the perturbation size. As the system converges, the new data are used to refine the shape of the $S$ surface in the vicinity of the stationary state. The same procedure was used to create and refine the $G$ function. Figure 4 shows an application of the $S$ function to suppress the oscillations of the autonomous system and to stabilize the unstable stationary state. The $S$ function was replaced by the $G$ function at $t=14500$ to alter the system output between the values of 0.2 and 0.3 . Only two
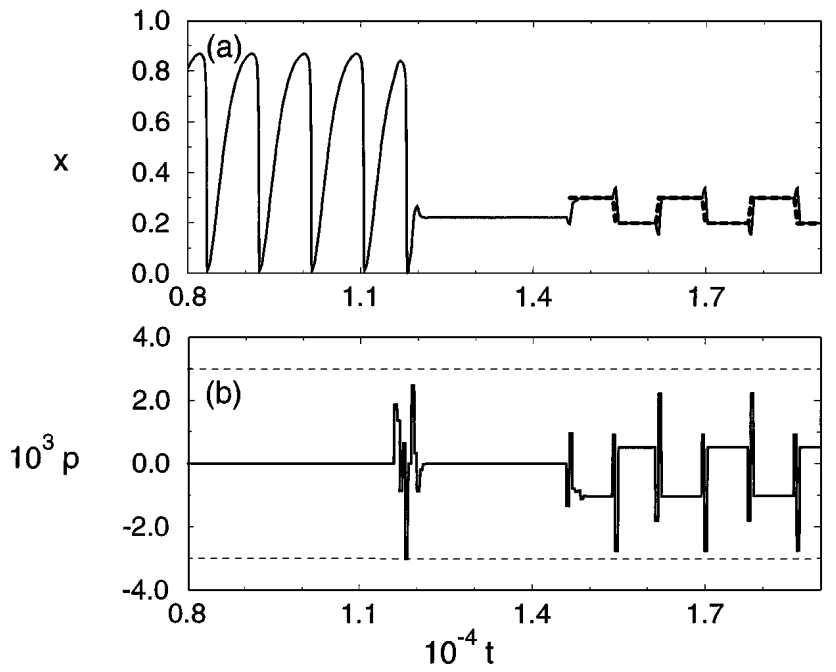

FIG. 4. (a) Stationary state stabilization and targeting objective states using seven-dimensional control surfaces $S$ and $G$. Broken line shows the objective states. (b) Applied perturbations, where dashed lines show the maximum allowed perturbation. iterations are required to move the system between these values of the unstable state.

The algorithms proposed here can readily be extended to include multiple observation and control channels by using a vector form of the $S$ or $G$ functions. Because the control laws are constructed directly from the time series, they are robust and convenient to implement in experimental settings. The number of unknown parameters for the control surface identification is generally higher than in the case of linear system identification and may therefore require larger data sets. The learning stage can be significantly decreased, however, by refining the control surface adaptively in the process of control. Because the control problem is formulated in terms of an invariant function, many well-developed techniques for prediction from nonlinear time series [19] can be used with the control algorithm.

We thank the National Science Foundation (Grant No. CHE-9531515), the Office of Naval Research (Grant No. N00014-95-1-0247), and the Petroleum Research Fund (Grant No. 29565-AC6) for supporting this research.

*To whom correspondence should be addressed.

[1] E. Ott, C. Grebogi, and J. A. Yorke, Phys. Rev. Lett. 64, 1196 (1990).

[2] W.L. Ditto, S. N. Rauseo, and M.L. Spano, Phys. Rev. Lett. 65, 3211 (1990).

[3] E. R. Hunt, Phys. Rev. Lett. 67, 1953 (1991).

[4] T. Carroll, I. Triandaf, I. B. Schwartz, and L. Pecora, Phys. Rev. A 46, 6189 (1992).

[5] R. Roy, T. W. Murphy, T. D. Maier, Z. Gills, and E. R. Hunt, Phys. Rev. Lett. 68, 1259 (1992).

[6] S. Bielawski, D. Derozeir, and P. Glorieux, Phys. Rev. A 47, R2492 (1993).

[7] V. Petrov, V. Gáspár, J. Masere, and K. Showalter, Nature (London) 361, 240 (1993).

[8] P. Parmananda, P. Sherard, R. W. Rollins, and H.D. Dewald, Phys. Rev. E 47, R3003 (1993).

[9] A. Garfinkel, M. L. Spano, W.L. Ditto, and J. N. Weiss, Science 257, 1230 (1992).

[10] S. J. Schiff, K. Jerger, D. H. Duong, T. Chang, M. L. Spano, and W. L. Ditto, Nature (London) 370, 615 (1994).

[11] T. Shinbrot, C. Grebogi, E. Ott, and J. A. Yorke, Nature (London) 363, 411 (1993).

[12] P. So and E. Ott, Phys. Rev. E 51, 2955 (1995).

[13] V. Petrov, E. Mihaliuk, S. K. Scott, and K. Showalter, Phys. Rev. E 51, 3988 (1995).

[14] V. Petrov, S. Metens, P. Borckmans, G. Dewel, and K. Showalter, Phys. Rev. Lett. 75, 2895 (1995).

[15] P. Gray and S. K. Scott, Chem. Eng. Sci. 39, 1087 (1984).

[16] Z. Gills, C. Iwata, R. Roy, I. B. Schwartz, and I. Triandaf, Phys. Rev. Lett. 69, 3169 (1992).

[17] V. Petrov, M. F. Crowley, and K. Showalter, Phys. Rev. Lett. 72, 2955 (1994).

[18] V. In, W. L. Ditto, and M. L. Spano, Phys. Rev. E 51, R2689 (1995).

[19] H. D. I. Abarbanel, R. Brown, J. J. Sidorowich, and L. S. Tsimring, Rev. Mod. Phys. 65, 1331 (1993). 